\title{
Open-Type Magnetically Shielded Room Combined With Square Cylinders Made of Magnetic and Conductive Materials for MRIs
}

\author{
Keita Yamazaki ${ }^{1}$, Shigetaka Hirosato ${ }^{1}$, Kiyotaka Kamata ${ }^{2}$, Kazuhiro Muramatsu ${ }^{3}$, Koichiro Kobayashi ${ }^{4}$, and \\ Akira Haga ${ }^{5}$ \\ ${ }^{1}$ Research and Development Institute, Takenaka Corporation, Chiba 270-1395, Japan \\ ${ }^{2}$ Department of Electric Control Engineering, Kagoshima National College of Technology, Kagoshima 899-5193, Japan \\ ${ }^{3}$ Department of Electrical and Electronic Engineering, Saga University, Saga 840-8502, Japan \\ ${ }^{4}$ Department of Welfare Engineering, Iwate University, Iwate 020-8551, Japan \\ ${ }^{5}$ Department of Electrical Engineering and Information Technology, Tohoku Gakuin University, Miyagi 985-8537, Japan
}

\begin{abstract}
To improve the comfort of hospital patients, we have developed a new open-type magnetically shielded room (MSR) for magnetic resonance imaging (MRI), constructed by stacking square cylinders composed of magnetic material on the inside and a conductive material on the outside. The magnetic cylinder shields against leakage of the magnetic field from the MRI, while the conductive outer cylinder prevents intrusion of the electromagnetic field from outside by waveguide attenuation. First, the optimal design of the magnetic cylinder was determined, using 3-D magnetic field analysis with a simple cubic MSR. Second, the validity of the analysis and the practical realization of the designed MSR were investigated using a small experimental model. Finally, from a design perspective and the standpoint of saving space, the optimal combination of electromagnetically shielded glass and conductive cylinders made of aluminum was studied by measuring the electromagnetic shielding effect. As the optimal design of an open-type MSR for an MRI, we have adopted a combination of single-layered electromagnetically shielded glass together with stacked cylinders. These cylinders are made of a square cylinder, 144 $\times 144 \mathrm{~mm}$ and $300 \mathrm{~mm}$ long, made of silicon steel (thickness $t=1 \mathrm{~mm}$ ) and divided into three parts along the axial direction, placed inside a second square cylinder made of aluminum, $150 \times 150 \mathrm{~mm}$ and $300 \mathrm{~mm}$ long $(t=3 \mathrm{~mm})$. This MSR construction technique creates an open, spacious feeling inside the facility and can be easily fabricated at low cost.
\end{abstract}

Index Terms-Electromagnetic field, leakage magnetic field, magnetic resonance imaging, multilayered magnetically shielded room, opening.

\section{INTRODUCTION}

I $\mathrm{N}$ order to reduce leakage flux density to less than $0.5 \mathrm{mT}$ outside the room [1] and shield against an electromagnetic field at high frequency during operation [2], a magnetic resonance imaging (MRI) scanner is usually installed in a magnetically shielded room (MSR) surrounded by a wall with magnetic and conductive layers. The small space enclosed by the wall causes patients a great deal of stress and discomfort because they are isolated from the operators and from the scenery outside. In order to improve the comfort of hospital patients, opentype MSRs have been designed for MRIs, using magnetic bars [3], canceling coils [4] instead of magnetic walls, and doublelayered electromagnetically shielded glass [5] instead of conductive walls. However, these MSRs cannot provide a sufficiently open feeling because of the lack of transparency and the optical moiré pattern of the double-layered glass. Moreover, electromagnetically shielded glass is expensive compared with the cost of magnetic and conductive walls.

We have therefore developed a new open-type MSR using a square cylinder made of a magnetic material, silicon steel, placed inside a second square cylinder made of a conductive material, aluminum, as shown in Fig. 1. Instead of a solid wall, there is a stack of hollow cylinders; this configuration creates an open feeling, the combination of aluminum and steel provides the shielding effect, and the construction also enables easy,

Digital Object Identifier 10.1109/TMAG.2008.2002519

${ }^{1}$ Color versions of one or more of the figures in this paper are available online at http://ieeexplore.ieee.org.

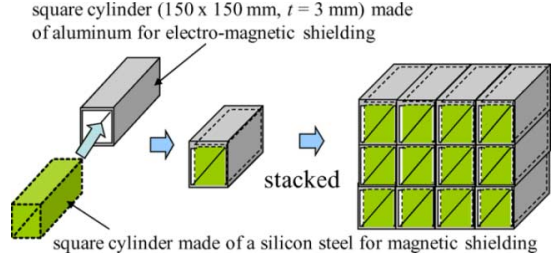

Fig. 1. A new open-type MSR for MRI using a combination of a square cylinder made of a magnetic material, silicon steel, placed inside a second square cylinder made of a conductive material, aluminum.

low-cost fabrication and removal. The size of the square cylinders is determined to make patients feel comfortable and unconfined. The conductive outside cylinder prevents the deterioration of the magnetic properties of the magnetic cylinder due to stress. The conductive cylinders also create an electromagnetic shield through waveguide attenuation. The leakage electromagnetic field through the gaps can be reduced by the electrical contact between the square aluminum cylinders. Therefore, the number of panes of shielded glass can be decreased, creating an even more spacious environment.

In this paper, the optimal design of an open-type MSR for MRIs using square cylinders made of a combination of magnetic and conductive materials, is developed using 3-D magnetic field analysis and experiments. First, the optimal design of the magnetic cylinder, including length, the number of divisions within the inner cylinders, and the width of the gap between the square cylinders, was determined using 3-D magnetic field analysis with a simple cubic MSR. Second, the validity of the analysis and the practical feasibility of the designed MSR were investigated using a small experimental model. Finally, from a design perspective and the standpoint of conserving space depending on the length of the cylinders, the optimal combina- 


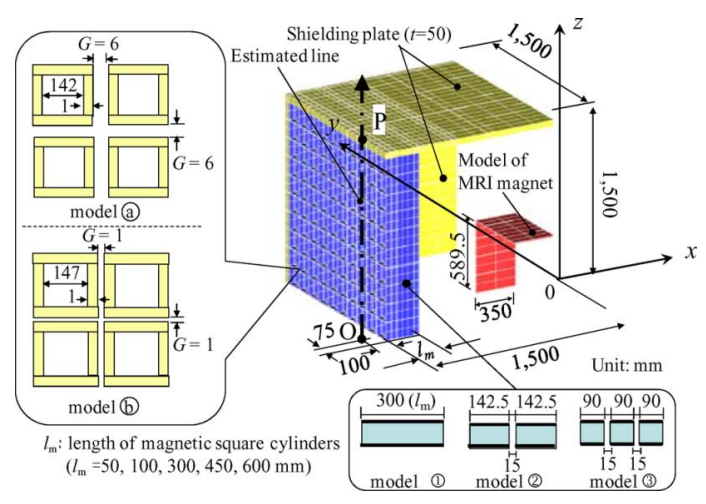

Fig. 2. Analyzed model (1/8 model).

tion of electromagnetically shielded glass and square cylinders made of aluminum was studied by measuring the electromagnetic shielding effect.

\section{OPtimal Design of MAGNeTiC CYlindERS}

\section{A. Model of Magnetically Shielded Room and Method of Analysis}

Fig. 2 shows the analyzed model of the MSR. Only an octant is described since the fields are symmetrical. Four sides of this MSR are composed of walls of magnetic material, silicon steel, and the opposite two sides are made of stacked cylinders, also made of silicon steel. The thickness of the magnetic walls is $50 \mathrm{~mm}$. The thickness of the magnetic cylinders is $1 \mathrm{~mm}$. The leakage flux densities (LFD) along line O-P were calculated under the conditions described below. The length $l_{m}$ of cylinders was changed to $50,100,300,450$, and $600 \mathrm{~mm}$. The gap $G$ between cylinders was changed to 1 and $6 \mathrm{~mm}$ in order to investigate the impact of leaving sufficient space for the conductive cylinder while retaining the magnetic shielding effect. Moreover, cylinders that were optionally divided into two and three parts by gaps of $15 \mathrm{~mm}$ along the axial direction are compared to one without a gap.

In magnetic field analysis, the 3-D finite-element method was applied, employing magnetic vector potential and a first-order brick edge element [4]. The space of the analyzed region was five times of that of the MSR in each direction and the Dirichlet boundary condition was imposed on the boundary. The relative permeability of silicon steel was assumed to be 3000 . The silicon steel was subdivided into two layers in the direction of thickness. The number of elements was 315900.

\section{B. Results and Discussion}

Fig. 3 shows the distribution of LFD depending on the length $l_{m}$ of the square cylinder for $G=1 \mathrm{~mm}$. We see a large reduction with increasing $l_{m}$ up to $300 \mathrm{~mm}$, which is around two times as long as the width of the opening. Longer cylinders would minimally improve the leakage, but would reduce the open feeling for the patients. Therefore, $l_{m}=300 \mathrm{~mm}$ is the optimal length.

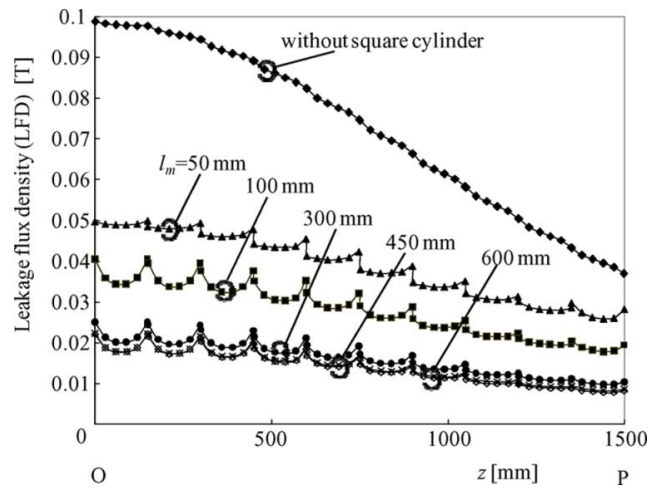

Fig. 3. Distribution of leakage flux densities versus length $l_{m}$ of the square cylinder $(G=1 \mathrm{~mm})$.

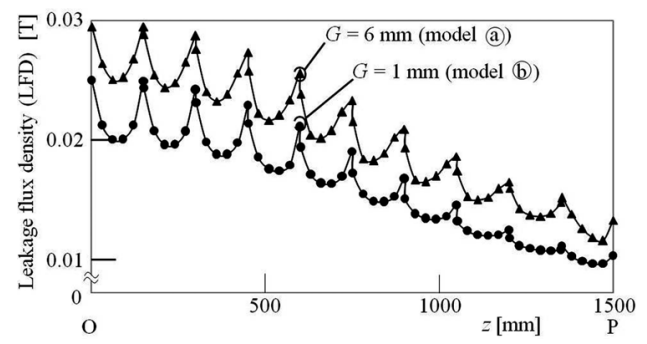

Fig. 4. Distribution of leakage flux densities versus width $G$ of the gap between the square cylinders $\left(l_{m}=300 \mathrm{~mm}\right)$.

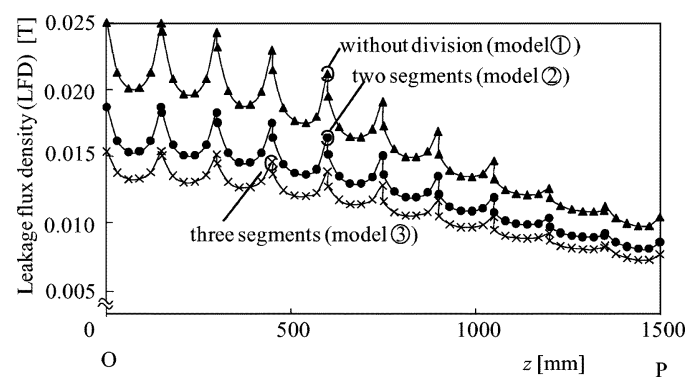

Fig. 5. Distribution of leakage flux densities versus number of divisions by gaps of $15 \mathrm{~mm}$ along axial direction $\left(G=1 \mathrm{~mm}, l_{m}=300 \mathrm{~mm}\right)$.

Fig. 4 shows the distribution of LFD depending on the width $G$ of the gap between cylinders for $l_{m}=300 \mathrm{~mm}$. An increased gap between magnetic cylinders due to the presence of the conductive layers is acceptable because the difference between the LFDs of gaps of 1 and $6 \mathrm{~mm}$ is not large compared to the difference between LFDs with and without magnetic cylinders.

Fig. 5 shows the LFD distribution depending on the number of divisions by the gaps of $15 \mathrm{~mm}$ along the axial direction for $l_{m}=300 \mathrm{~mm}$ and $G=1 \mathrm{~mm}$. The LFD of a cylinder divided into three segments is lower than that of cylinders without gaps or divided into two segments. Fig. 6 shows the distribution of magnetic flux density vectors inside the magnetic material of the square cylinder, depending on the number of divisions. Especially near the $x$ axis, in (b) and (c), the magnetic flux reaching the outer layer is reduced due to the magnetic resistance of the gaps, resulting in the small LFD. 


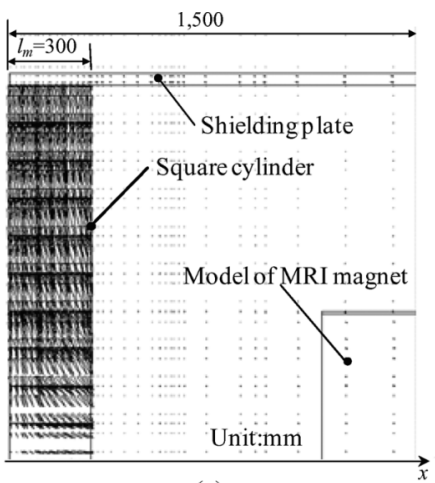

(a)

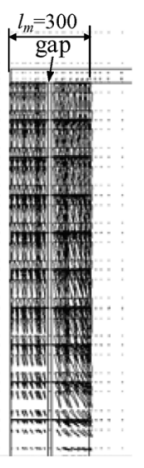

(b)

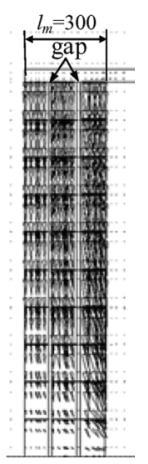

(c)
Fig. 6. Distribution of magnetic flux vectors in the magnetic materials of the square cylinders depending on the number of divisions along the axial direction by gaps of $15 \mathrm{~mm}$ (a) without division (model (1)), (b) two segments (model (2)), and (c) three segments (model (3)).

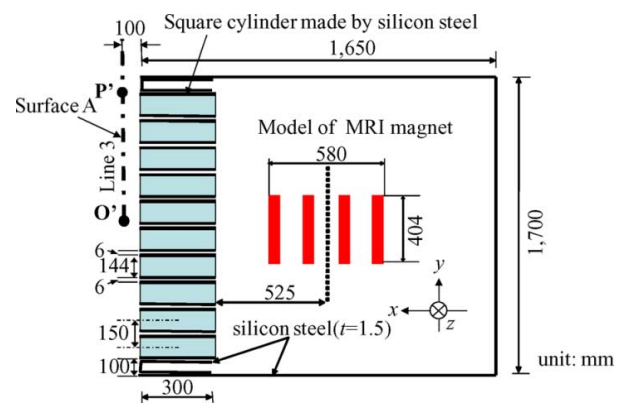

Fig. 7. Small model of magnetically shielded room and MRI magnet.

\section{EXPERIMENTAL VERIFICATION}

\section{A. Experimental Small Model and Methods of Measurement and Analysis}

In this section, the validity of the calculation and the practical realization of the MSR designed in this paper are investigated using a small experimental model, shown in Fig. 7. Sixty square cylinders composed of aluminum $(t=3 \mathrm{~mm})$ and silicon steel $(t=1 \mathrm{~mm})$ were stacked on the opening of the model MSR. The current of each of the four circular coils that served as the MRI model was 1485 AT and it flowed in the same direction in each coil in order to generate a large leakage flux. The cylinders were divided into three segments by gaps of 15 or $30 \mathrm{~mm}$, along the axial direction, or were not divided.

In order to confirm the effect of the dividing the cylinders, the distributions of LFD were measured and calculated for no gap or 15- or 30-mm gaps. The measurements were carried out using a three-axial fluxgate magnetometer. In this analysis, the relative permeability of the silicon steel of the cylinder and wall was set to be 1000 due to the smaller flux densities in the silicon steel than those reported in Section II. The number of elements was 171500 .

\section{B. Results and Discussion}

Fig. 8 shows the measured distributions of LFD along line $\mathrm{O}^{\prime}-\mathrm{P}^{\prime}$ in Fig. 7. The LFD of a cylinder divided into three segments is lower than that of a cylinder without gaps. The LFD of a cylinder divided into three segments by gaps of $15 \mathrm{~mm}$ is almost the same as that of a cylinder with $30-\mathrm{mm}$ gaps. From

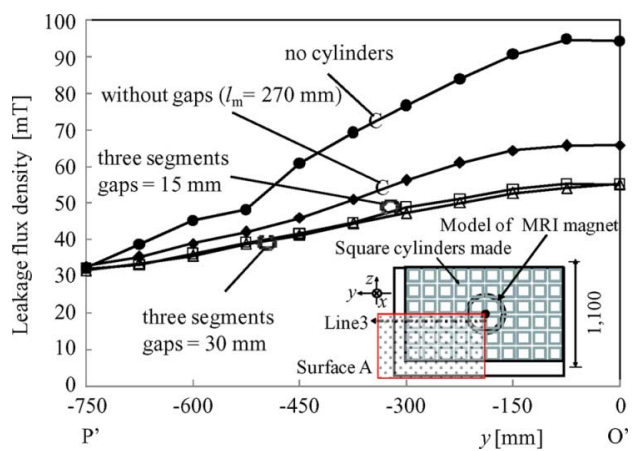

Fig. 8. Measured leakage flux density distributions along line 3.

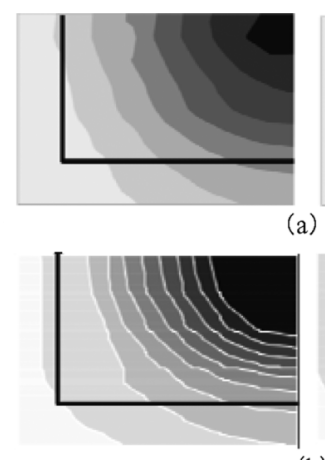

(i)

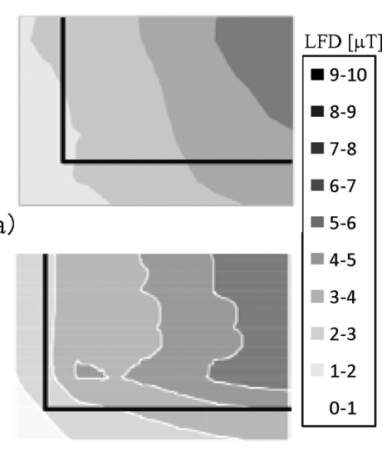

(ii)
Fig. 9. Comparison of the contours of the magnitude of leakage flux density at the surface A obtained from calculation and measurements: (a) measured, (b) calculated, (i) without square cylinders, and (ii) with square cylinders divided into three segments.

these measurements, the length of the gap dividing the magnetic cylinder into three segments was chosen to be $15 \mathrm{~mm}$.

Fig. 9 compares the contours of the magnitude of LFD at surface A, shown in Figs. 7 and 8, obtained from calculation and measurement. This figure shows that the tendency of the calculation is in good agreement with the measurements.

\section{Optimal COMBINATION OF ElECTROMAGNETICALly SHIELDED GLASS AND SQUARE CYLINDERS FOR} Electromagnetic SHIELDING EFFECT AT High FreQUENCIES

\section{A. Model of Wall Patterns and Method of Measurement}

In order to operate MRIs, the electromagnetic shielding effect $S E$, as defined by the following equation, is required to be 100 $\mathrm{dB}$ at frequencies from 60 to $180 \mathrm{MHz}$ [2]:

$$
S E[\mathrm{~dB}]=L_{1}[\mathrm{~dB} \mu \mathrm{V}]-L_{2}[\mathrm{~dB} \mu \mathrm{V}]
$$

where $L_{1}$ and $L_{2}$ are the signals at the receiving antenna without and with a shielding wall placed between the transmitting and receiving antennas.

First, in order to understand the $S E$ of a wall composed only of conductive cylinders, the $S E$ (in decibels) of waveguide attenuation was estimated by (2) and (3) [6] as follows:

$$
\begin{aligned}
S E & =8.69 \times(2 \pi f / C) \times \sqrt{\left(f_{c} / f\right) \times 2-1} \times l_{c} \\
f_{c} & =C /(2 A)
\end{aligned}
$$




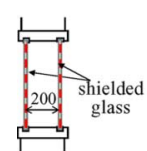

(a)

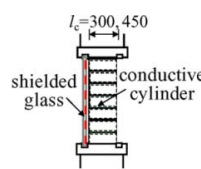

(b)

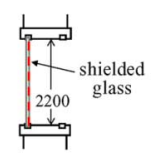

(c)

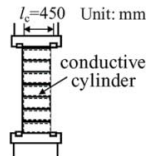

(d)
Fig. 10. Measured models of wall patterns composed of electromagnetically shielded glass (\#60) and conductive cylinders made of aluminum: (a) doublelayered glasses (Model A), (b) single-layered glass and cylinders (Model B), (c) single-layered glass only (Model C), and (d) cylinders only (Model D).

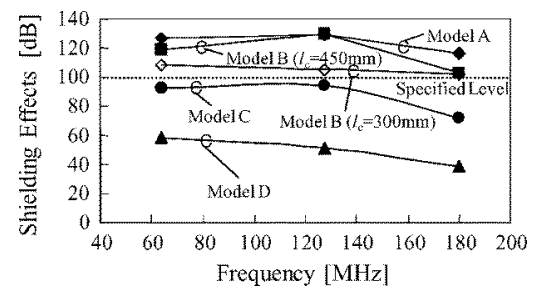

Fig. 11. Measured electromagnetic shielding effect of various wall patterns.

where $f$ and $f_{c}$ are the frequency of electromagnetic field and the cutoff frequency, respectively; $C$ is the velocity of light; and $l_{c}$ and $A$ are the length and edge length of a sectional area of the waveguide. In order to satisfy the specific $S E$ of $100 \mathrm{~dB}$ at $f=100 \mathrm{MHz}, C=3 \times 10^{8} \mathrm{~m} / \mathrm{s}$, and $A=150 \mathrm{~mm}, l_{c}$ should be about $550 \mathrm{~mm}$. However, this length $l_{c}=550 \mathrm{~mm}$ does not create a very open feeling. Therefore, the optimal combination of electromagnetically shielded glass and shorter square cylinders with $l_{c}=300$ and $450 \mathrm{~mm}$ was investigated.

On the other hand, the edges of the bending sheet of magnetic material should be connected to each other by welding in order to retain the electric conductivity between them and enable waveguide attenuation. But the shielding effect to reduce the leakage flux from the MRI does not remain, due to the deterioration of magnetic properties caused by the welding. Therefore in these measurements, the magnetic cylinder without welding is neglected, and another cylinder made of conductive material is placed outside the magnetic cylinder. Aluminum was selected as the conductive material from the viewpoint of weight, cost, and manufacturing accuracy on the plane surface and edge angle in comparison with copper and stainless steel. Moreover, in order to retain electrical contact between the square cylinders for reducing leakage electromagnetic field through the gap, a thin film of nickel was plated onto the surface of the cylinders.

Fig. 10 shows measured models of wall patterns composed of electromagnetically shielded glass (\#60) and square conductive cylinders. Model A used ordinary double-layered shielded glasses. Model B is the proposed combination of single-layered glass and conductive cylinders. The thickness and opening of the aluminum square cylinders are $3 \mathrm{~mm}$ and $150 \times 150 \mathrm{~mm}$, respectively. For purposes of comparison, the measurements were also carried out using single-layered glass only (Model C) and conductive cylinders only (Model D).

The electromagnetic shielding effects $S E$ at frequencies from 60 to $180 \mathrm{MHz}$ were measured in an experimental electromagnetically shielded room. The wall pattern models were placed into a $1500 \times 2200 \mathrm{~mm}$ opening in the solid shielded wall, and were placed between horizontal biconical antennas for transmitting and receiving, each of which was set $1000 \mathrm{~mm}$ away from the wall [7].

\section{B. Results and Discussion}

Fig. 11 shows the measured $S E$ s of various wall pattern models. Models C and D cannot achieve the specified level of $100 \mathrm{~dB}$. On the other hand, both Models A and B with $l_{c}=300 \mathrm{~mm}$ can satisfy the specified level of $100 \mathrm{~dB}$ level. However, Model A does not provide a sufficiently open feeling, due to the optical moiré pattern of the double layered glass, and it is expensive. In Model B, these disadvantages are overcome.

\section{CONCLUSION}

As the optimal design of an open-type MSR for an MRI, we have adopted a combination of single-layered electromagnetically shielded glass together with stacked square cylinders. Each square cylinder is composed of two square cylinders. The inner of these, $144 \times 144 \mathrm{~mm}$ and $300 \mathrm{~mm}$ long, is made of silicon steel $(t=1 \mathrm{~mm})$ divided into three parts by the gaps of $15 \mathrm{~mm}$ along the axial direction to shield against leakage of the magnetic field from the MRI. It is placed inside a second square cylinder made of aluminum, $150 \times 150 \mathrm{~mm}$ and $300 \mathrm{~mm}$ long $(t=3 \mathrm{~mm})$, that shields the electromagnetic field from outside by waveguide attenuation. This MSR developed by a combination of cylinders and a single-layer glass effectively shields against leakage of the magnetic field from the MRI, satisfies specified electromagnetic shielding effect levels for MRIs operating at frequencies from 60 to $180 \mathrm{MHz}$, and creates an unconfined, open feeling for patients by reducing the amount of electromagnetically shielded glass. In addition, the construction method of stacking the cylinders is easy and inexpensive.

\section{REFERENCES}

[1] ICNIRP, "Guideline to limits of exposure to static magnetic fields," Health Phys., vol. 66, pp. 113-122, 1994.

[2] Technical Publication, Signa HDx 3.0T Pre-Installation Copyright 2006 by General Electric Company, Inc..

[3] T. Saito, "The opened-type magnetic shielding," in Abstract INTERMAG 2003 Conf., 2003, no. CR-03.

[4] S. Hirosato et al., "Open type of magnetically shielded room combined with canceling coil for magnetic resonance imaging," IEEE Trans. Magn., vol. 42, no. 10, pp. 3542-3544, Oct. 2006.

[5] J. L. N. Violette, D. R. J. White, and M. F. Violette, Electromagnetic Compatibility Handbook. New York: Van Nostrand Reinhold, 1987, pp. 372-376.

[6] F. E. Terman, Electronic and Radio Engineering, 4th ed. New York: McGraw-Hill, 1955, p. 128.

[7] Attenuation Measurement for Enclosures, Electromagnetic Shielding, for Electronic Test Purposes, Method of, MIL-STD-285, 1956.

Manuscript received March 03, 2008. Current version published December 17, 2008. Corresponding author: K. Yamazaki (e-mail: yamazaki.keita@takenaka.co.jp). 